PROCEEDINGS OF THE

AMERICAN MATHEMATICAL SOCIETY

Volume 131, Number 9, Pages 2721-2725

S 0002-9939(03)07119-3

Article electronically published on April 21, 2003

\title{
TRACES OF CONVEX DOMAINS
}

\author{
CEZAR JOIŢA
}

(Communicated by Mohan Ramachandran)

\begin{abstract}
Diederich and Ohsawa proved that in $\mathbb{P}^{5}$ there exists a locally hyperconvex, Stein open subset which is not hyperconvex. In this paper we generalize their results.
\end{abstract}

\section{INTRODUCTION}

In 2] Diederich and Ohsawa proved that if $M$ is a complex manifold and $N$ is a complex submanifold, then any locally hyperconvex, Stein open subset of $N$ is the trace of a locally hyperconvex, Stein open subset of $M$. In [4 it was proved that if $Y$ is a closed complex subspace of $X$ and $Y$ is Stein, then $Y$ has a Stein neighborhood. Also, it has been proved in $[5]$ that if $Y$ is hyperconvex, then $Y$ has a hyperconvex neighborhood.

Using the methods of Demailly [1 we will set up a general framework for the above theorems and we will generalize Diederich and Ohsawa's results for reduced complex spaces.

\section{THE RESULTS}

If $M$ is a topological space we will denote by $\mathcal{C}(M)$ the set of continuous real functions defined on $M$, and by $\operatorname{Open}(M)$ the set of open subsets of $M$.

Let $\mathcal{A}$ be the class of reduced complex spaces and let $\mathcal{B} \subset \mathcal{A}$ be such that for every $M \in \mathcal{B}$, Open $(M) \subset \mathcal{B}$. We assume also that for every $x \in M,\{x\} \in \mathcal{B}$. For each $M \in \mathcal{B}$ we consider a subset $\mathcal{P}(M)$ of $\mathcal{C}(M)$ such that the following conditions are satisfied:

1) For every $M \in \mathcal{B}$ and $U$ an open subset of $M$, if $\phi \in \mathcal{P}(M)$, then $\phi_{\mid U} \in \mathcal{P}(U)$. Furthermore $\mathcal{P}_{\mid \operatorname{Open}(M)}$ is a subsheaf of sets of $\mathcal{C}_{\mid \operatorname{Open}(M)}$ and if $M$ is just a point, then $\mathcal{P}(M)=\mathbb{R}$.

2) For every $f, g \in \mathcal{P}(M)$, a real number $a>0, U$ an open subset of $\mathbb{R}$ containing $g(M)$ and $\chi: U \rightarrow \mathbb{R}$ a smooth, convex, and non-decreasing function, we have af $+\chi \circ g \in \mathcal{P}(M)$ and $\max \{f, g\} \in \mathcal{P}(M)$.

3) For every $N \subset M$ a closed subspace, $N, M \in \mathcal{B}$, every continuous function $\lambda: N \rightarrow(0, \infty)$, and every $f \in \mathcal{P}(N)$ there exists an open neighborhood $V \subset M$ of $N$ and $\tilde{f} \in \mathcal{P}(V)$ such that $\left|\tilde{f}_{\mid V}-f\right|<\lambda$.

4) For every $N \subset M$ a closed subspace, $N, M \in \mathcal{B}$, there exists an open neighborhood $V$ of $N$ and a continuous function $f: V \rightarrow[-\infty, \infty)$ such that $f^{-1}(-\infty)=N$

Received by the editors March 19, 2001.

2000 Mathematics Subject Classification. Primary 32C15, 32E10, 32Q28.

(C)2003 American Mathematical Society 
and $f$ has the following property: for every $x \in V$ and every $\phi \in \mathcal{P}_{x}$ there exist $k>0$ and an open neighborhood $U$ of $x$, contained both in $V$ and in the domain of $\phi$, such that $f+k \phi \in \mathcal{P}(U \backslash N)$. A function with this property will be called almost $\mathcal{P}$.

Definition 1. Let $M \in \mathcal{B}$

1. $M$ is said to be $\mathcal{P}$-complete if there exists $\phi \in \mathcal{P}(M)$ such that for every $c \in \mathbb{R},\{x \in M: \phi(x)<c\} \subset \subset M$.

2. $M$ is said to be hyper $\mathcal{P}$-complete if there exists $\phi \in \mathcal{P}(M), \phi: M \rightarrow(-\infty, 0)$, such that for every $c<0,\{x \in M: \phi(x)<c\} \subset \subset M$.

3. An open subset $D$ of $M$ is said to be locally hyper $\mathcal{P}$-complete if for every $x \in \partial D$ there exists an open neighborhood $B$ of $x$ such that $B \cap D$ is hyper $\mathcal{P}_{-}$ complete.

Observation. It follows from the properties of $\mathcal{P}$ that every point of $M \in \mathcal{B}$ has a hyper $\mathcal{P}$-complete neighborhood.

We consider $N, M \in \mathcal{B}, N$ a closed subspace of $M$. The proofs of the following two propositions are similar to the proof of Theorem 1 in [1. Only the proof of Proposition 2 will be given here.

Proposition 1. If $N$ is $\mathcal{P}$-complete, then $N$ has a $\mathcal{P}$-complete neighborhood in $M$.

Proposition 2. If $N$ is hyper $\mathcal{P}$-complete, then $N$ has a hyper $\mathcal{P}$-complete neighborhood in $M$.

Proof. Let $U$ be an open neighborhood of $N$ and $v: U \rightarrow[-\infty, \infty)$ a continuous function such that $v^{-1}(-\infty)=N$ and $v$ is almost $\mathcal{P}$ on $U$. Shrinking $U$ we may suppose that there exists $\phi \in \mathcal{P}(U)$ such that $\phi<0$ and for every $c \in \mathbb{R},\{x \in N$ : $\phi(x)<c\} \subset \subset N$. Let $W$ be an open subset of $M$ such that $\partial W \backslash N \subset U, N \subset W$ and for every $c<0,\{x \in \bar{W}: \phi(x) \leq c\}$ is compact.

Let $\tilde{v}=v+\chi \circ \phi$ where $\chi:(-\infty, 0) \rightarrow \mathbb{R}$ is a smooth, convex and increasing function. If $\chi$ increases fast enough $\tilde{v} \in \mathcal{P}(W \backslash N)$. To see that one sets $F_{n}:=$ $\phi^{-1}\left(\left[\frac{-1}{n}, \frac{-1}{n+1}\right]\right)$ and $F_{0}:=\phi^{-1}(-\infty,-1]$. For every $j \in \mathbb{N}, F_{j}$ is compact and therefore there exists a neighborhood $U_{j}$ of $F_{j}$ and $k_{j}>0$ such that $v+k_{j} \phi \in$ $\mathcal{P}\left(U_{j} \backslash N\right)$. We then require that $\chi_{[[-1 / n,-1 / n+1]}^{\prime}>k_{j}$. The condition $\tilde{v} \in \mathcal{P}(W \backslash N)$ is a local condition and $\bigcup F_{j} \supset W$. On a neighborhood of $F_{j}, \tilde{v}=v+k_{j} \phi+\chi_{j} \circ \phi$ where $\chi_{j}(t)=\chi(t)-k_{j} t$ is a convex and increasing function on a neighborhood of $\phi\left(F_{j}\right)$. This implies that $\tilde{v} \in \mathcal{P}(W \backslash N)$. In the same way we can choose $\chi$ such that $\tilde{v}_{\mid \partial W \backslash N}>0$.

We set $V:=\{x \in W: \tilde{v}(x)<0\}$. Then $V \supset N$ and $\psi:=\max \{\phi, \tilde{v}\}$ is a negative exhaustion for $V$. Also, since $\psi=\phi$ in a neighborhood of $N$, we have $\psi \in \mathcal{P}(V)$.

Proposition 3. If $D$ is an open, $\mathcal{P}$-complete, and locally hyper $\mathcal{P}$-complete subset of $N$, then there exists an open, $\mathcal{P}$-complete, and locally hyper $\mathcal{P}$-complete subset $\Omega$ of $M$ such that $\Omega \cap N=D$.

Proof. We denote by $\partial D$ the boundary of $D$ in $N$. For every $x \in \partial D$ we consider an open neighborhood $Q_{x}$ of $x$ in $N$ such that $W_{x}:=Q_{x} \cap D$ is hyper $\mathcal{P}$-complete.

Let $\left\{Q_{j}: j \in \mathbb{N}\right\}$ be a countable subset of $\left\{Q_{x}: x \in \partial D\right\}$ such that $\bigcup Q_{j} \supset \partial D$. Using Proposition 2 we choose $\widetilde{W}_{j}$ open hyper $\mathcal{P}$-complete subsets of $M$ such that 
$\widetilde{W}_{j} \cap N=W_{j}$ and we set $\widetilde{W}=\bigcup \widetilde{W}_{j}$. Let $D_{1}$ be an open subset of $D$ such that $\bar{D}_{1} \subset D$ and $\left(D \backslash \bar{D}_{1}\right) \subset \widetilde{W}$. For every $j \in \mathbb{N}$ let $R_{j}$ be an open subset of $M$ such that $\bar{R}_{j} \cap N \subset Q_{j}, \bigcup R_{j} \supset \partial D, \bar{R}_{j} \cap D_{1}=\emptyset$ and $\left\{R_{j}\right\}$ is locally finite. For each $z \in D \backslash D_{1}$ we choose an open neighborhood $I_{z}$ in $M$ such that $I_{z} \cap N \subset D$ and for each $j \in \mathbb{N}$ we have:

if $z \in \bar{R}_{j}$, then $I_{z} \subset \widetilde{W}_{j}$,

if $z \notin \bar{R}_{j}$, then $I_{z} \cap \bar{R}_{j}=\emptyset$.

This is possible because $\left\{R_{j}\right\}$ is locally finite and $\bar{R}_{j} \cap D \subset \widetilde{W}_{j}$. Note that because $\left\{R_{j}\right\}$ is locally finite $\bigcup \bar{R}_{j}$ is closed. For $z \in D_{1}$ we choose an open neighborhood $J_{z}$ in $M$ such that $J_{z} \cap\left(\bigcup \bar{R}_{j}\right)=\emptyset$ and $J_{z} \cap N \subset D$.

Put

$$
U:=\left(\bigcup_{z \in D \backslash D_{1}} I_{z}\right) \cup\left(\bigcup_{z \in D_{1}} J_{z}\right) .
$$

Then $U$ is open in $M$ and $U \cap N=D$. Also for every $j \in \mathbb{N},\left(R_{j} \cap U\right) \subset\left(\widetilde{W}_{j} \cap U\right)$. Using Proposition 1 we choose a $\mathcal{P}$-complete open subset $U_{1} \subset U$ of $U$ such that $U_{1} \cap N=D$ and $\partial U_{1} \backslash N \subset U$. Let $\phi \in \mathcal{P}\left(U_{1}\right)$ be an exhaustion function and $v: U_{1} \rightarrow[-\infty, \infty)$ an almost $\mathcal{P}$ function such that $v^{-1}(-\infty)=D$. We can assume of course that $\phi>1$. Set $h=v+\chi \circ \phi$ where $\chi: \mathbb{R} \rightarrow \mathbb{R}$ is a smooth, convex and increasing function such that $h \in \mathcal{P}\left(U_{1} \backslash D\right)$. We define $\Omega:=\left\{x \in U_{1}: h(x)<0\right\}$. Then $\Omega$ is $\mathcal{P}$-complete since $\max \left\{\phi,\left(1-e^{h}\right)^{-1}\right\}$ is an exhaustion.

$\Omega$ is also hyper $\mathcal{P}$-complete. Indeed: if $x \in \partial \Omega \backslash N$, it follows directly from the definition that $\Omega$ is hyper $\mathcal{P}$-complete around $x$. If $x \in \partial \Omega \cap N=\partial D$, we choose $j \in \mathbb{N}$ such that $x \in R_{j}$ and let $B:=R_{j} \cup \tilde{W}_{j}$. Then $B$ is an open neighborhood of $x$ and $B \cap \Omega=\tilde{W}_{j} \cap \Omega$. If $\rho: \tilde{W}_{j} \rightarrow(-\infty, 0)$ is an exhaustion function for $\tilde{W}_{j}$ and $\rho \in \mathcal{P}\left(\tilde{W}_{j}\right)$, then $\psi:=\max \{h, \rho\}$ is a bounded exhaustion function for $B$ and $\psi \in \mathcal{P}(U)$.

Two examples. I) $\mathcal{B}=\mathcal{A}$ and, for a reduced complex space $X, \mathcal{P}(X)=$ the set of strictly plurisubharmonic functions. It follows from [1] that $\mathcal{P}$ satisfies all the required properties.

In this case Proposition 1 is the Main Theorem in [4] and Proposition 2 is Theorem 4 in [5]. Proposition 3 becomes:

Proposition 4. Let $Y$ be a complex subspace of $X$. If $D$ is an open, Stein, locally hyperconvex subset of $X$, then there exists an open, Stein, locally hyperconvex subset $\Omega$ of $X$ such that $\Omega \cap Y=D$.

It was proved in 2 that for every $n \geq 5$ there exists an open subset $\Omega$ of $\mathbb{P}^{n}$ which is Stein, locally hyperconvex but not hyperconvex.

If $Y$ is a projective algebraic variety of dimension $n \geq 5$ let $\pi: Y \rightarrow \mathbb{P}^{n}$ be a proper, surjective and finite holomorphic map and let $\Omega_{1}:=\pi^{-1}(\Omega)$. Since $\pi$ is finite, $\Omega_{1}$ is Stein and locally hyperconvex (see in this sense [5]).

Suppose $\Omega_{1}$ is hyperconvex. Let $\phi: \Omega_{1} \rightarrow(-\infty, 0)$ be a plurisubharmonic exhaustion function and let $\pi_{*} \phi$ be the unique continuous function that outside the branching set satisfies: $\pi_{*} \phi\left(x^{\prime}\right)=\sum_{\pi(x)=x^{\prime}} \phi(x)$.

By Varouchas [6], $\pi_{*} \phi$ is plurisubharmonic. If $p$ is the maximal number of points in the fiber of $\pi$ and $\epsilon>0$, then $\left\{\pi_{*} \phi \leq-\epsilon\right\} \subset \pi\left(\left\{\phi \leq \frac{-\epsilon}{p}\right\}\right)$. Therefore $\pi_{*} \phi$ is a bounded plurisubharmonic exhaustion function for $\Omega$. But a Stein domain that 
has a bounded plurisubharmonic exhaustion function is hyperconvex and this is a contradiction. Combining this last observation and Proposition 4 we obtain:

Theorem 1. Let $X$ be a complex space. If $X$ has a complex subspace $Y$ which is a projective algebraic variety with $\operatorname{dim}(Y) \geq 5$, then there exists an open Stein subset $\Omega \subset X$ which is locally hyperconvex but not hyperconvex.

II) Let $(X, \omega)$ be a Kähler manifold and set $\mathcal{B}:=\bigcup\{\operatorname{Open}(Y): Y$ is a closed submanifold of $X\}$. Every $M \in \mathcal{B}$ is a Kähler manifold with the induced metric from $X$. Definition 2 and Proposition 5 are due to $\mathrm{H}$. Wu 7 .

Definition 2. 1) Let $M$ be a Kähler manifold, $x \in M$ and $G$ the hermitian inner product on $T_{x} M$ given by the Kähler metric. A set of $q$ vectors $\left\{Z_{1}, Z_{2}, \ldots, Z_{q}\right\}$ is $\epsilon$-normal, for $\epsilon>0$, if $\left|G\left(Z_{i}, Z_{j}\right)-\delta_{i j}\right|<\epsilon$ for $i, j=1,2, \ldots, q$.

2) If $f$ is a continuous function defined near $x$ and $L$ is a 1-dimensional complex submanifold of $M$ passing though $x$ we choose a coordinate system $\left\{z_{1}, z_{2}, \ldots, z_{n}\right\}$ such that $z_{i}(x)=0, i=1, \ldots, n$, and such that near $x, L=\left\{z_{2}=z_{3}=\ldots=z_{n}=0\right\}$. Furthermore, assume $\left|\partial / \partial z_{1}\right|(x)=1$. Then we define

$$
P f(x, L)=\liminf _{r \rightarrow 0} \frac{2}{\pi r^{2}}\left(\int_{0}^{2 \pi} f\left(r e^{i \theta}, 0, \ldots, 0\right) d \theta-2 \pi f(0)\right) .
$$

If $Z \in T_{x} M$, also define $\operatorname{Pf}(x, Z)=|Z|^{2} \inf _{L} \operatorname{Pf}(x, L)$ where $L$ runs through all the 1-dimensional complex submanifolds of $M$ tangent to $\operatorname{span}_{\mathbb{R}}\{Z, J Z\}$ and defined near $x$.

3) If $U$ is an open subset of $M$ we define $\Psi(q ; U)$ to be those continuous functions $f$ defined on $U$ with the following property: for every $x_{0} \in U$ there exists a neighborhood $W$ of $x_{0}$ and positive constants $\epsilon$ and $\eta$ such that if $x \in W$ and $\left\{Z_{1}, \ldots, Z_{q}\right\}$ is an $\epsilon$-orthonormal set in $T_{x} M$, then

$$
\sum_{j=1}^{q} \operatorname{Pf}\left(x, Z_{j}\right) \geq \eta .
$$

If $f$ is a $C^{2}$ function we denote by $L f$ the Levi form of $f$.

Proposition 5. On a Kähler manifold $M$ the class $\Psi_{q}(M)$ enjoys the following properties:

a) A real-valued $C^{2}$ function, $f$, belongs to $\Psi_{q}(M)$ if and only if for each set of vector fields $\left\{Z_{1}, \ldots, Z_{q}\right\}$ which are orthonormal with respect to $G$ we have: $\sum_{i=1}^{q} L f\left(Z_{i}, Z_{j}\right)>0$.

b) $\Psi_{q}(M)$ is a cone in the space of continuous functions, i.e., if $f_{1}, f_{2}$ are in $\Psi_{q}(M)$, then so is any positive combination thereof.

c) $\Psi_{q}(M)$ has the maximum-closure property, i.e., if $f_{1}, f_{2}$ are in $\Psi_{q}(M)$, then so is $\max \left\{f_{1}, f_{2}\right\}$.

d) $C^{\infty} \cap \Psi_{q}(M)$ is dense in $\Psi_{q}(M)$ in the $C^{o}$ topology.

Then $\mathcal{P}:=\Psi_{q}$ satisfies properties 1), 2) and 4). $\mathcal{P}$ also satisfies 3 ). This follows from the density of $C^{\infty} \cap \Psi_{q}(M)$ in $\Psi_{q}(M)$ and from the next proposition:

Proposition 6. Let $M$ be an m-dimensional Kähler manifold, $N \subset M$ a closed $n$-dimensional complex submanifold, and $\phi \in \Psi_{q}(N) \cap C^{\infty}$. Then there exist a neighborhood $V$ of $N$ in $M$ and $\tilde{\phi} \in \Psi_{q}(V) \cap C^{\infty}$ such that $\tilde{\phi}_{\mid Y}=\phi$. 
Proof. We consider $\phi^{\prime}$ an arbitrary $C^{\infty}$ extension of $\phi$ to a neighborhood of $N$ and $\left\{\Omega_{\lambda}, z_{\lambda}\right\}$ a locally finite covering of $N$ with coordinate patches $z_{\lambda}: \Omega_{\lambda} \rightarrow \mathbb{C}^{m}$ in which $N \cap \Omega_{\lambda}$ is given by $z_{\lambda}^{\prime}=\left(z_{\lambda, n+1}, \ldots, z_{\lambda, m}\right)=0$. Let $\left\{\theta_{\lambda}\right\}$ be $C^{\infty}$ functions with compact support in $\Omega_{\lambda}$ such that $\sum \theta_{\lambda}=1$ on $N$. Set

$$
\tilde{\phi}=\phi^{\prime}(x)+\sum \theta_{\lambda} \log \left(1+\epsilon_{\lambda}^{-1}\left|z_{\lambda}^{\prime}\right|^{2}\right) .
$$

Then $\tilde{\phi}_{\mid N}=\phi$ and for $x \in N \cap \operatorname{supp}\left(\theta_{\mu}\right), L \tilde{\phi} \geq L \phi^{\prime}+\theta_{\mu} \epsilon_{\mu}{ }^{-1} L\left|z_{\mu}^{\prime}\right|^{2}$ and therefore if we choose $\left\{\epsilon_{\lambda}\right\}$ to be small enough it follows that $\tilde{\phi} \in \Psi_{q}(V)$ for some neighborhood $V$ of $N$.

\section{AKNOWLEDGEMENTS}

I am very grateful to Professor Mihnea Colţoiu for inspiring this work and to Professor Terrence Napier for many useful discussions and suggestions.

\section{REFERENCES}

[1] J. P. Demailly, Cohomology of q-convex spaces in top degrees, Math. Z. 204 (2) (1990), 283295. MR 91e:32014

[2] K. Diederich; T. Ohasawa, On pseudoconvex domains in $\mathbb{P}^{n}$, Tokyo J. Math. 21 (1998), 353358. MR 99k:32024

[3] T. Ohsawa, A Stein domain with smooth boundary which has a product structure, Publ. Res. Inst. Math. Sci. 18 (1982), 1185-1186. MR 84i:32022

[4] Y. T. Siu, Every Stein subvariety admits a Stein neighborhood, Invent. Math. 38 (1976/77), 89-100. MR 55:8407

[5] V. Vâjâitu, On locally hyperconvex morphisms, C. R. Acad. Sci. Paris Ser. I Math. 322 (9) (1996), 823-828. MR 97b:32014

[6] J. Varouchas, Stabilité de la classe des variétés käleriénnes par certains morphismes propres, Invent. Math. 77 (1) (1984), 117-127. MR 86a:32026

[7] H. Wu, On certain Kähler manifolds which are q-complete, Complex analysis of several variables (Madison, Wis., 1982), Proc. Sympos. Pure Math., vol. 41., pp. 253-276. MR 85j:32031

Institute of Mathematics of the Romanian Academy, P.O. Box 1-764, RO-70700, Bucharest, Romania

Current address: Department of Mathematics, Lehigh University, Bethlehem, Pennsylvania 18015

E-mail address: cej3@lehigh.edu 